\title{
Parallel Computation for Electronic Waves in Quantum Corrals
}

\author{
HENRY K. HARBURY* and WOLFGANG POROD ${ }^{\dagger}$ \\ University of Notre Dame, Notre Dame, IN 46556
}

\begin{abstract}
Recent scanning tunneling microscopy (STM) studies on the (111) faces of noble metals have directly imaged electronic surface-confined states and dramatic standing-wave patterns have been observed $[1,2]$. We solve for the local density of electronic states in these "leaky" quantum corral confinement structures using a coherent elastic scattering theory. We seek solutions of the two-dimensional Schrödinger equation compatible with non-reflecting boundary conditions which asymptotically satisfy the Sommerfeld radiation condition $[11,14]$. The large matrices generated by the discretization of realistic quantum corral structures require the use of sparse matrix methods. In addition, a parallel finite element solution was undertaken using the message passing interface standard (MPI) and the Portable, Extensible, Toolkit for Scientific Computation (PETSc) [5] for an efficient computational solution on both distributed and shared memory architectures. Our calculations reveal excellent agreement with the reported experimental $d I / d V$ STM data.
\end{abstract}

Keywords: Parallel computing; finite element method; open-boundary systems; non-reflecting boundary conditions; quantum corrals.

\section{INTRODUCTION}

Recent scanning tunneling microscopy (STM) differential conductance studies in the vicinity of defects and step edges on $\mathrm{Cu}(111)$ at $T=4.2 \mathrm{~K}$ [1-3] and on $\mathrm{Au}(111)$ and $\mathrm{Ag}(111)$ at room temperature [4,5] have directly imaged the dramatic standing wave patterns of the electronic surface-confined states [6]. We solve for the local density of electronic states (LDOS) in quantum corral structures using a coherent elastic scattering theory. We seek solutions of the twodimensional Schrödinger equation compatible with Sommerfeld radiation conditions $[3,4]$ in these "leaky" confinement structures. We obtain excellent agreement with the reported experimental STM differential conductance studies. Our results are consistent with the STM junction current model of Tersoff and Hamann [7] and Lang [8], as extended by Davis et al. [9] to the case of the 2DEG formed by surfacestate confinement, in which the differential conductance, $d I / d V$, is a measure of the surface LDOS.

The LDOS is evaluated from the analytic Green function obtained from the system Hamiltonian compatible with an outward Sommerfeld radiation condition that is consistent with open-boundary electronic scattering. The numerical technique implements nonreflecting boundary conditions (NRBC) on an artificial boundary enclosing the scattering domain and is

\footnotetext{
* Director of Science Computing Facilities, 202-A Nieuwland, College of Science, University of Notre Dame, Notre Dame, IN 46556.

$\doteqdot$ Department of Electrical Engineering, 268 Fitzpatrick, College of Engineering, University of Notre Dame, Notre Dame, IN 46556.
} 
compatible with standard finite element methods (FEM) [10,11]. We discuss our parallel FEM approach using the message passing interface standard (MPI) [12] and the Portable, Extensible, Toolkit for Scientific Computation (PETSc) [13]. The processor decomposition is designed to reduce the communication required for the assembly of the sparse global matrix for an efficient solution on both distributed and shared memory architectures.

\section{MODEL SYSTEM}

An electronic scattering state, $\psi_{E}$, with energy $E$ must satisfy the Schrödinger equation for the system Hamiltonian, $\mathrm{H} \psi_{E}=E \psi_{E}$, where

$$
-\frac{\hbar^{2}}{2} \nabla \cdot\left[\frac{1}{m^{*}} \nabla \psi_{E}(\mathbf{r})\right]+V(\mathbf{r}) \psi_{E}(\mathbf{r})=E \psi_{E}(\mathbf{r}) .
$$

The potential term, $V(\mathbf{r})$, encompasses the elastic scattering centers, which are modeled as finite-height potential barriers. The local density of states (LDOS) is obtained from the diagonal of the Green function,

$\operatorname{LDOS}(\mathbf{r} ; E)=-\frac{1}{\pi} \operatorname{Im}\left[\mathrm{G}^{+}(\mathbf{r}, \mathbf{r} ; E)\right]$, where $\mathbf{G}=(E-\mathbf{H})^{-1}$,

Following the work of Bayliss and coworkers [14], we formulate approximate non-reflecting boundary conditions on an artificial boundary at a radius $R_{0}$ which encloses the scattering domain; the potential is assumed constant outside the artificial boundary and is taken as the zero reference. A boundary constraint is imposed which restricts the solutions to the class of wavefunctions which are compatible with an outward Sommerfeld radiation condition $[15,16]$, $\lim _{r \rightarrow \infty} r^{(d-1) / 2}\left({ }_{r} \psi-i k \psi\right)=0$, where $d$ is the system dimensionality. In the two-dimensional case, the scattered part of the wavefunction may be expanded in the form $\psi_{\text {scatt }} \alpha e^{i k r} \sum_{j=0}^{\infty} f_{j}(\theta) /(k r)^{j+\frac{1}{2}}$. A set of linear operators, $B_{m}$, are constructed such that each $m^{\text {th }}$ higher order term is eliminated by the $B_{m}$ operation on the far-field expansion, $B_{m} \psi_{s c a t t} \Gamma=O\left(R_{0}^{-\frac{4 m+1}{2}}\right)$.
The boundary constraint is exact in the limit of $R_{0} \rightarrow \infty$ and has a radially decaying truncation error on the boundary. The set of operators are therefore defined as $B_{0}=1, B_{m}=\left(\partial_{r}-i k+\alpha_{m} / r\right) B_{m-1}$, where $\alpha_{m}$ is a constant chosen to eliminate the $m^{\text {th }}$ term of the expansion. The subsequent formulation of the Green function using a finite element basis and recast into matrix notation has the form:

$$
\mathbf{G}=[E \mathbf{M}-(\mathbf{T}+\mathbf{V}+\mathbf{C})]^{-1},
$$

where $\mathbf{T}$ and $\mathbf{V}$ contain the kinetic and potential energy matrix elements, respectively, $\mathbf{C}$ contains the matrix elements which result from the NRBC $[10,11]$, and the matrix elements of $M$ result from the nonorthogonal finite element basis set.

The large systems generated by the FEM discretization of realistic quantum corral structures can benefit from parallel sparse matrix storage and parallel computation techniques. Using triangular mesh elements and standard node number reordering methods, narrowly banded systems are generated. By distributing the independent local element calculations across processors and likewise distributing the sparse global system matrix, assembly of the linear system can be performed with minimal interprocess communication. Schematically shown in Fig. 1 is the processor domain decomposition of the finite element mesh. Each box superimposed over the schematic finite element domain on the left encloses the local elements assigned to each processor. Since all the processors simultaneously initialize with the element data required for their respective subdomain computations, the only required interprocessor communication is for global matrix assembly. As is schematically shown in the figure, the storage of the sparse global matrix is distributed across the processors such that each computes most of its locally stored global matrix elements. The required communication is therefore due to the assembly of the processor boundary contributions. This communication penalty can be further reduced through the use of non-blocking sends to overlap the FEM integral computations with the global matrix assembly. The local NRBC matrix elements, $\mathbf{C}$, do not disturb the sparse matrix structure and require no additional communication overhead.

There has also been much recent effort to develop efficient parallel Krylov subspace (KSP) type iterative 


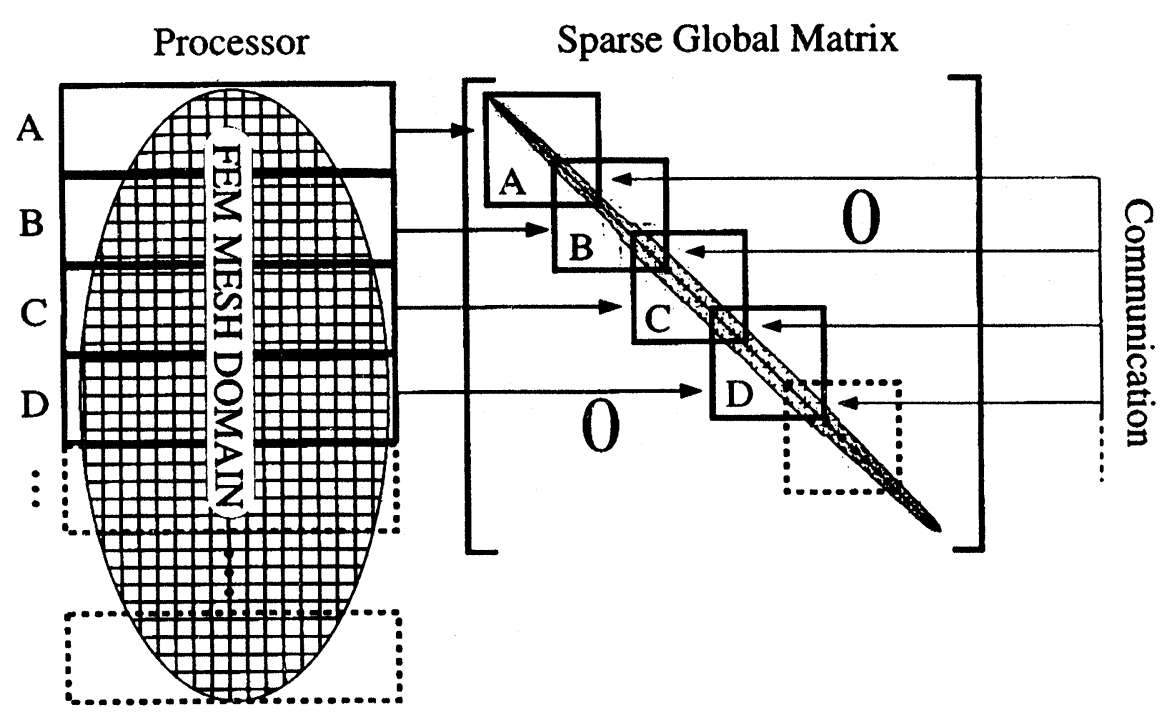

FIGURE 1 Schematic diagram for the parallel domain decomposition of the finite element mesh domain. The element contributions from each processor region on the left assembles into the distributed sparse global matrix on the right.

Communication is only required to assemble the processor boundary contributions

solvers. In particular, the Portable, Extensible, Toolkit for Scientific Computation (PETSc) [13] library, implemented with the Message Passing Interface (MPI) standard [12], provides a simple programmer interface to parallel sparse vector and matrix datastructures and many powerful KSP linear and non-linear solvers. While insulating the programmer from the details of the underlying communication, the PETSc library provides access to the full richness of the MPI standard while maintaining as much as possible the "single system view" of traditional sequential codes. The portable library is available for most common hardware platforms from workstations to supercomputers. Since it is built using installed MPI libraries, it takes advantage of any site dependent MPI optimizations for its underlying hardware communications.

\section{RESULTS}

Using our elastic scattering theory, we calculate the LDOS for quantum corral structures based on the geometries reported in Refs. [1] and [3]. Each numerical scattering domain was discretized by a non-uniform triangular mesh extending beyond each $\mathrm{Fe}$ adatom corral. The Fe adatoms, discretized and modeled as hard-wall finite potential barriers, $E_{B}$, of $1.52 \AA$ diameter, are positioned equidistant from the $2.55 \AA$ spaced nearest-neighbor hexagonal $\mathrm{Cu}(111)$ sites. We found that one set of parameters, $\mathrm{m}^{*}=$ $0.361 m_{0}$ and $E_{B}=2.5 e \mathrm{~V}$, was able to reproduce the available experimental data, and it was used for all our elastic scattering calculations. The effective mass is comparable to the $m * / m_{0}=0.38 \pm 0.02$ reported by Crommie et al. [2]. We use an approximate band edge energy of $0.43 \mathrm{eV}$ below $E_{F}$, a value comparable to the $0.44 \pm 0.01 \mathrm{eV}$ band edge reported in Ref. [2]. Shown in Fig. 2 is a typical two-dimenstional image of the LDOS for the $76 \mathrm{Fe}$ Adatom oval quantum corral. The standing wave patterns are clearly visible and compare well to the reported experimental STM differential conductance data [3]. 


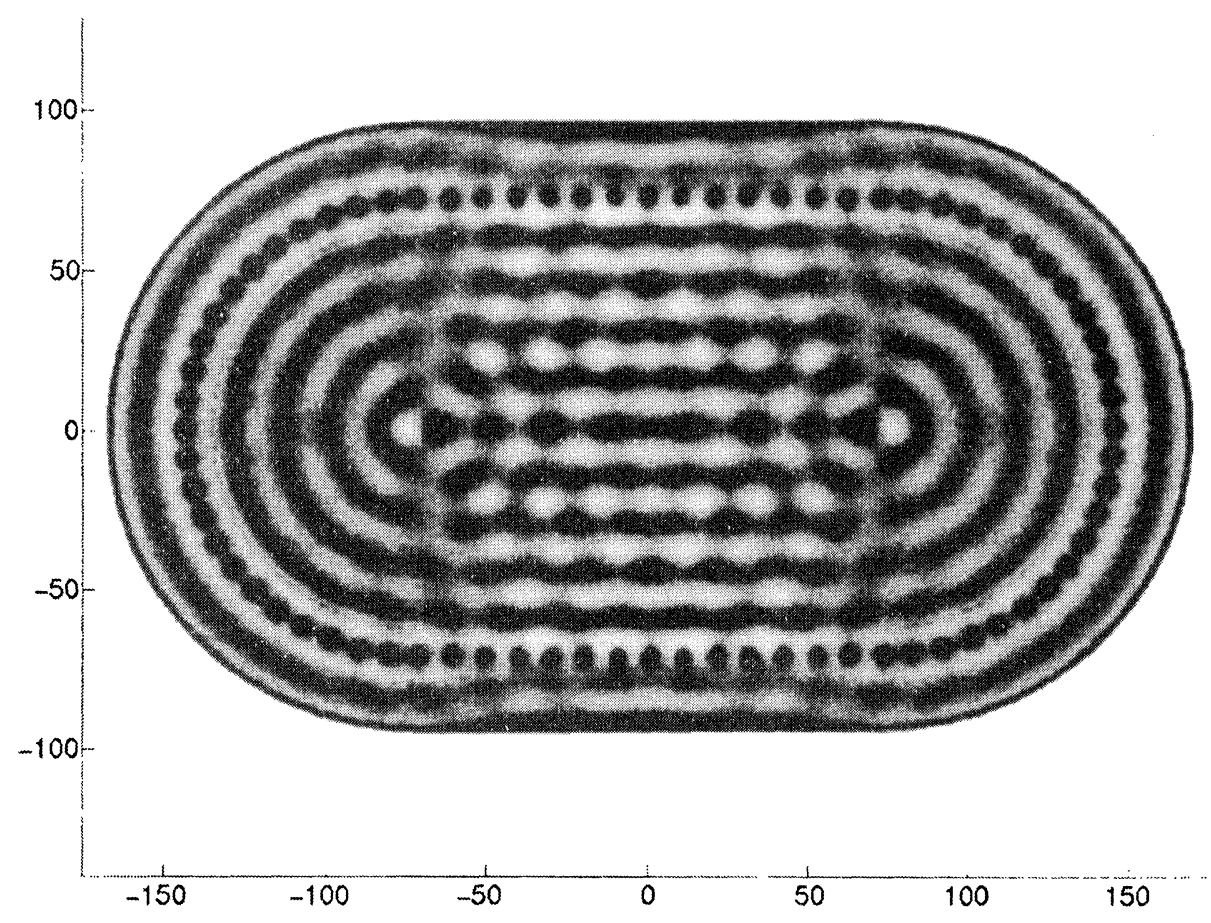

FIGURE 2 Image of the theoretical elastic scattering calculation of the LDOS for the $76 \mathrm{Fe}$ adatom oval "stadium" corral for $E=0.45 \mathrm{eV}$. Excellent agreement is achieved with the reported STM $d I / d V$ image in Ref. [3]

\section{CONCLUSION}

In summary, we have presented an elastic scattering theory for the electronic states in quantum corrals and our approach to a parallel FEM numerical solution using the PETSc [13] libraries. The parallel processor decomposition of the FEM mesh and the global solution matrix are distributed such that little interprocessor communication is necessary during the assembly stage. The overall excellent agreement of our results with available STM data on $\mathrm{Cu}(111)$ leads us to conclude that the $\mathrm{Fe}$ adatoms act as elastic scattering centers to the surface-confined electrons, and that there is no significant coupling between the 2DEG and bulk states.

\section{Acknowledgements}

We thank Dr. Craig S. Lent and Dr. R. Kent Smith for numerous helpful discussions, and gratefully acknowledge partial financial support by AFOSR, ARPA, and ONR.

\section{References}

[1] M. F. Crommie, C. P. Lutz, and D. M. Eigler, Science 262 , 218 (1993).

[2] M. F. Crommie, C. P. Lutz, and D. M. Eigler, Nature 363, 524 (1993).

[3] E. J. Heller, M. F. Crommie, C. P. Lutz, and D. M. Eigler, Nature 369, 464 (1994).

[4] Y. Hasegawa and Ph. Avouris, Phys. Rev. Lett. 71, 1071 (1993).

[5] Ph. Avouris, I.-W. Lyo, R. E. Walkup, and Y. Hasegawa, J. Vac. Sci. Technol. B May/June (1994); Ph. Avouris and I.-W. Lyo, Science 264, 942 (1994).

[6] S. D. Kevan and R. H. Gaylord, Phys. Rev. B 36, 5809 (1987).

[7] J. Tersoff and D. R. Hamann, Phys. Rev. B 31, 805 (1985); Phys. Rev. Lett. 50, 1998 (1983).

[8] N. D. Lang, Phys. Rev. B 34, 5947 (1986); ibid. 56, 1164 (1986); Phys. Rev. Lett. 55, 230 (1985).

[9] L. C. Davis, M. P. Everson, R. C. Jaklevic, and W. Shen, Phys. Rev. B 43, 3821 (1991).

[10] H. K. Harbury and W. Porod, J. Appl. Phys. 75, 5142 (1994).

[11] H. K. Harbury, W. Porod, and R. K. Smith, in Proceedings of the Third International Workshop on Computational Electronics, ed. by S. M. Goodnick, pp. 153 (Portland, OR, May 1994).

[12] William Gropp, Ewing Lusk, Anthony Skjellum, Using MPI, Portable Parallel Programming with the Message-Passing Interface, The MIT Press, 1995. 
[13] Barry Smith, William Gropp, and Lois Curfman McInnes, PETSc 2.0 libraries, Mathematics and Computer Science Division, Argonne National Laboratory.

URL:<http: //www.mcs.anl.gov/petsc/petsc.html>

[14] A. Bayliss, M. Gunzburger, and E. Turkel, SIAM J. Appl. Math. 42, 430 (1982).

[15] A. Sommerfeld, Lectures on Theoretical Physics(Academic Press, New York, 1964).

[16] D. Givoli, J. Comput. Phys. 94, 1 (1991).

\section{Biographies}

Henry Harbury is the Director of Science Computing Facilities in the College of Science at the University of Notre Dame. His research interests include computational modeling of solid state material physics and electronic transport in mesoscopic and microscopic systems. He is a member of the APS and the IEEE.

Wolfgang Porod is Professor of Electrical Engineering at the University of Notre Dame. His research interests include computational solid state electronics and the physics of computation. He is a member of the APS and a senior member of the IEEE. 

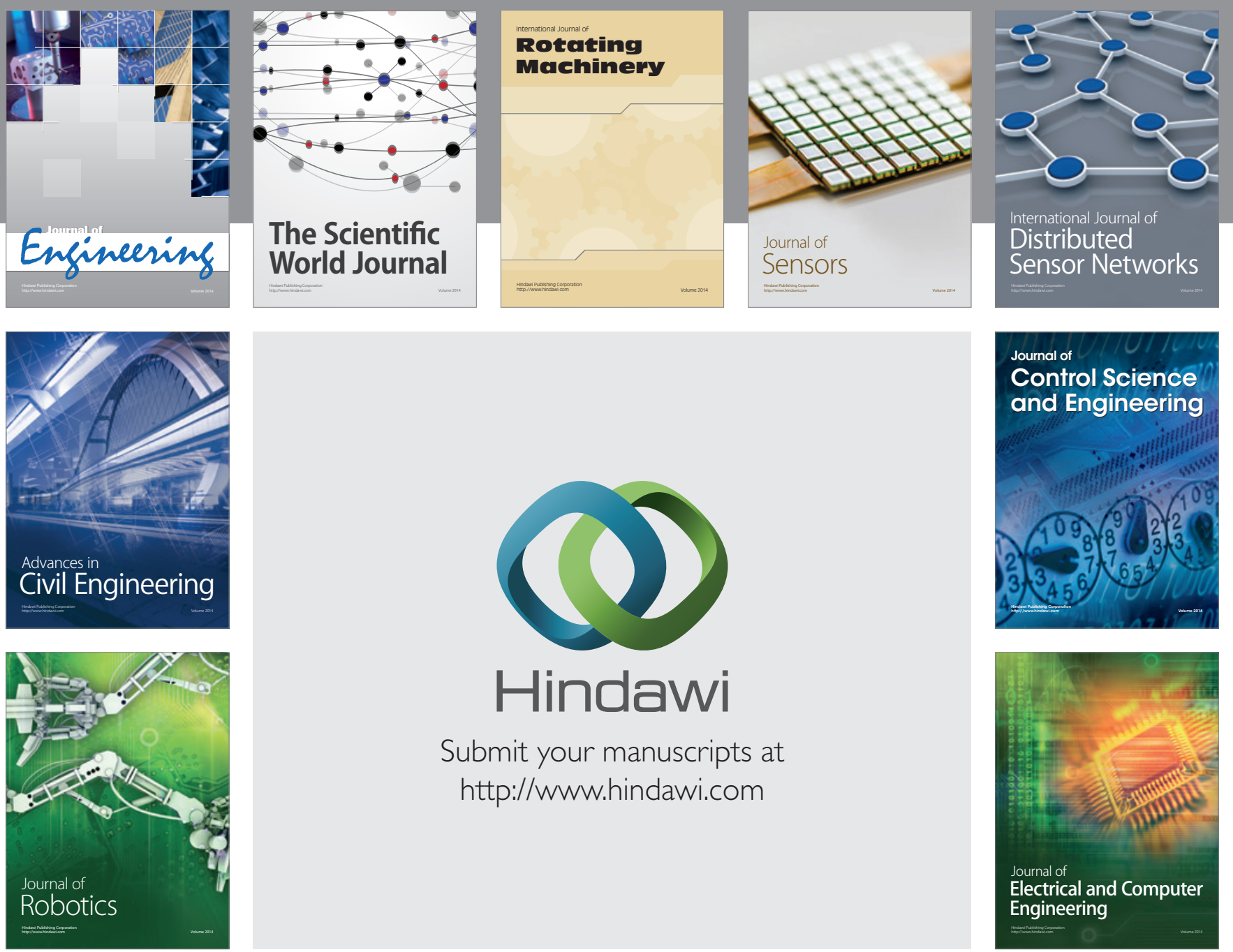

Submit your manuscripts at

http://www.hindawi.com
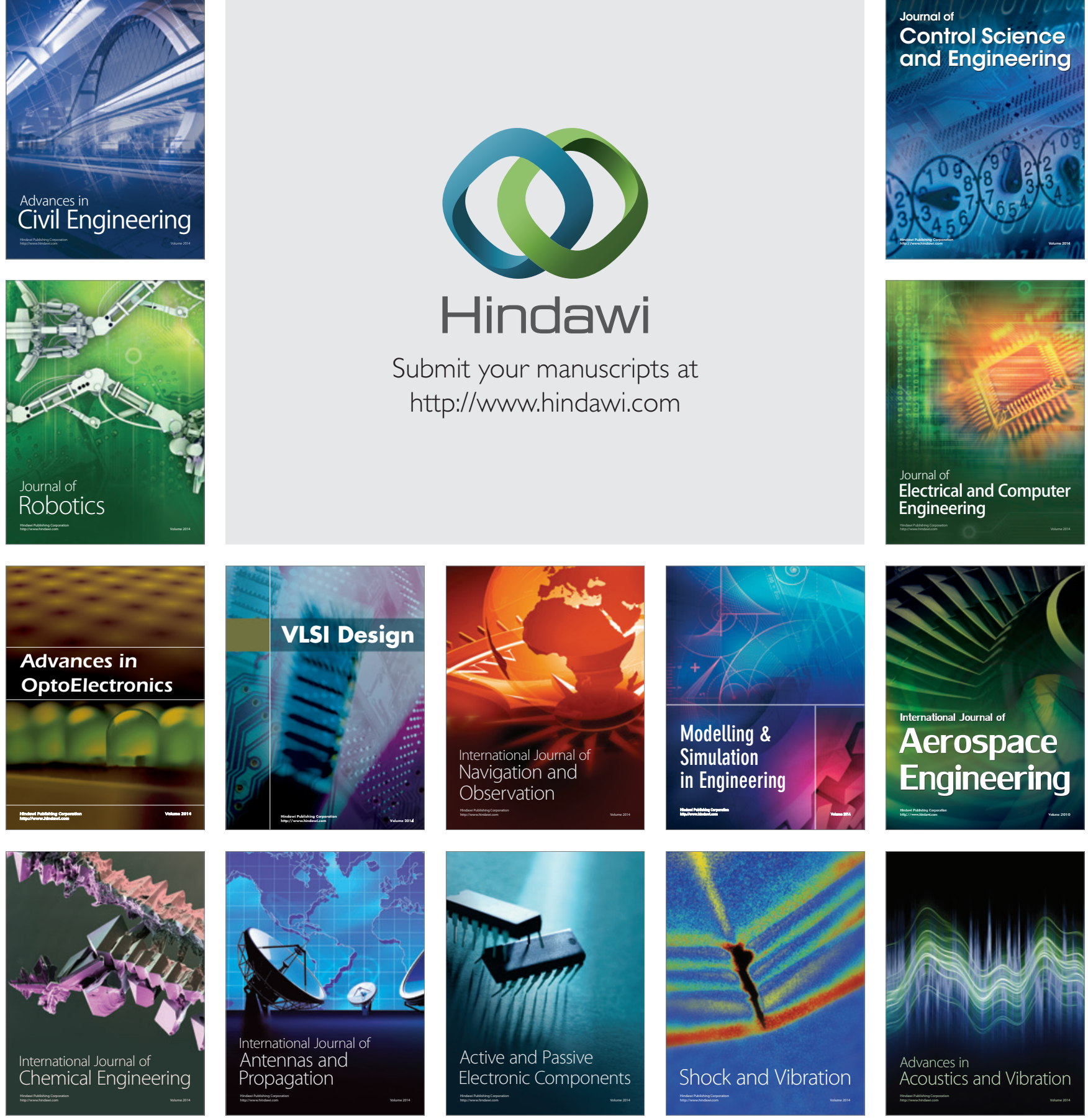